\title{
A Review on Four Degrees of Freedom (DOF) Wireless Robotic Arm based on LabVIEW Software
}

\author{
Mrugank Arekar \\ Student, Department of ECE \\ Babaria Institute of Technology, Varnama
}

\author{
Hetal M. Pathak, PhD \\ Professor, Department of ECE \\ Babaria Institute of Technology, Varnama
}

\begin{abstract}
The aim of this paper is to thoroughly elaborate designing, development and to implement steps involved to make a superior four degrees of freedom (DoF) robotic ARM which is more organized and having low expenditure. The proposed robotic ARM is designed with four degrees of freedom to perform various associated tasks, such as material handling, shifting which can serve as an assistant for industries and, moreover this robotic arm can be useful in hazardous calamities such as earthquakes to identify and rescue people stuck under the debris. The robotic ARM is built with the number of servomotors that perform ARM movements concurrently. The controlling action of robotic ARM are managed through graphical programming language G; Lab VIEW (Laboratory Virtual Instrument Engineering Workbench) which was developed by National Instruments. Lab VIEW communicates with the robotic arm using appropriate movement angles through Arduino that drives the servomotors having the capability of varying position. At last, using wireless communication the robotic arm movements are controlled and implemented in an organized manner which follows UART protocol.
\end{abstract}

\section{Keywords}

Robotic arm, Degrees of freedom, Servo motor, LabVIEW, Arduino UNO, RF Zigbee, Serial Communication.

\section{INTRODUCTION}

The use of robotic arms has increased intensely in the past decade. We are able to actuate the robot for specific and exact needs with more efficient and accurate sensors [1]. For the last few decades, unmanned robots-vehicles are becoming very popular and common in R\&D, Industries home and military organizations. There are many advantages of these robots as compared to human contributions to those application areas. One of the most important things about these robots is that they have the capability to perform their action remotely in the field, where human cannot enter and do the activity without any risks to human lives which shows a great impact on robots [3].

Robots can be described into two types: a service robot and an industrial robotic. A service robot operates usually as a semi-auto or fully autonomously to perform services useful to the humans and equipment, excluding manufacturing operations.

Whereas, compared to service robot, the Industrial robot is an automatically controlled and multipurpose programmable in three or more axis. Industrial robots are basically designed to shift material, parts, tools, and equipment, or specialized devices through variable programmed motions to perform a variety of tasks. An industrial robot system comprises of various devices or sensors required for the robot to perform its tasks as well as sequencing or monitoring communication interfaces [6]

\section{MOTIVATION AND OBJECTIVE}

The technologies in the industry are growing sharply in this recent era. As growing day by day, more and more electronic products developed are more advanced compared to past years. Today, robotics is a far rapidly growing field, as the technology advances due to continuous research and new technology replacing the older ones. Robotics serves various practical purposes, whether domestically, industrially or militarily. Wireless Robotic arms can be used in any situation and for any purpose as they can be used in dangerous environments (including bomb detection and de-activation), manufacturing processes, or where humans cannot reach easily.

The main objective is to build a robotic arm which would be less in weight and obtain high accuracy by implementing a serial communication UART protocol connected to the RF Zigbee module which makes the robotic arm travel at a high range. The proposed robotic arm is designed to be useful in the applications where the humans cannot reach easily such as hazardous calamities, industrial automation etc.

\section{HARDWARE DESCRIPTION}

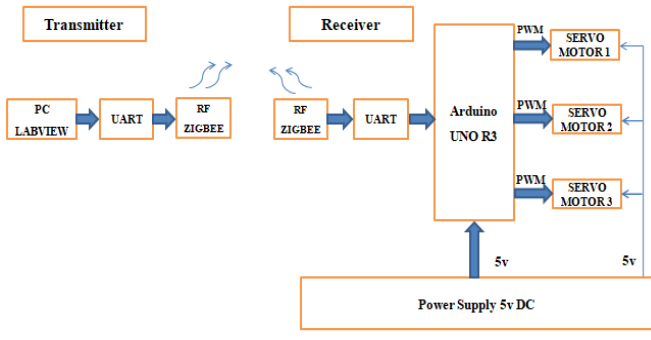

Figure 1: Block diagram of the system for controlling four degrees of freedom (DOF) wireless robotic arm

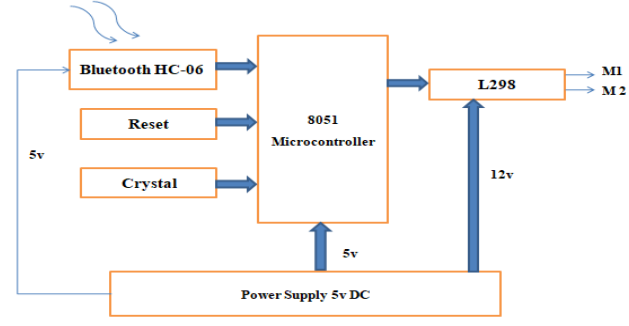

Figure 2: Block diagram for controlling the mobility of the robotic arm 
The block diagram of the system for controlling four degrees of freedom(DOF) wireless robotic arm using LAB VIEW software contains following components: Arduino UNO R3 Microcontroller, Power Supply 5V DC, RF Zigbee, UART Module, Servo Motor (V3003), Pulse Width Modulation(PWM), Force Sensitive Resistor, Data Glove, Flex Sensor, Accelerometer, PIR (Passive Infrared) Sensor, Robotic arm Vehicle, Bluetooth Module, BT Voice Control for Android (Mobile Application), NI(National Instruments) Lab View (Software) and Proteus Design Suite.

The mobility of the robotic arm is controlled by using a Bluetooth module connected to a mobile application named "BT voice control for Arduino" which works on the principle of voice process. As per the instructions given the robotic arm mobility is achieved

Circuitry contains a Bluetooth Module HC-06, Reset key, Crystal, 8051 Microcontroller, L298 board and power supply $5 \mathrm{v}$ DC.

\section{FLOWCHART OF THE SYSTEM}

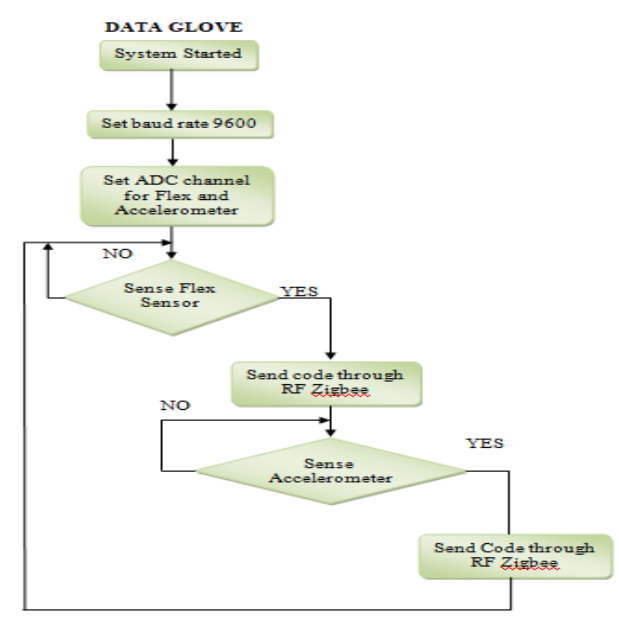

Figure 3: Flowchart of the Dataglove

\section{(i) For Data Glove}

$\rightarrow$ For the operation of the data glove, we first initiate the system and set baud rate at $9600 \mathrm{bits} / \mathrm{sec}$.

$\rightarrow$ The second step is to set the ADC channel for flex and accelerometer sensor.

$\rightarrow$ First, it senses flex sensor and then sends code through RF Zigbee and if successful the data is forwarded and if not then it the cycle repeats.

$\rightarrow$ Same step is applied for the accelerometer.

$\rightarrow$ The data glove is fitted to one of the hands and the controlling of the robotic arm is achieved by programming it through Arduino.

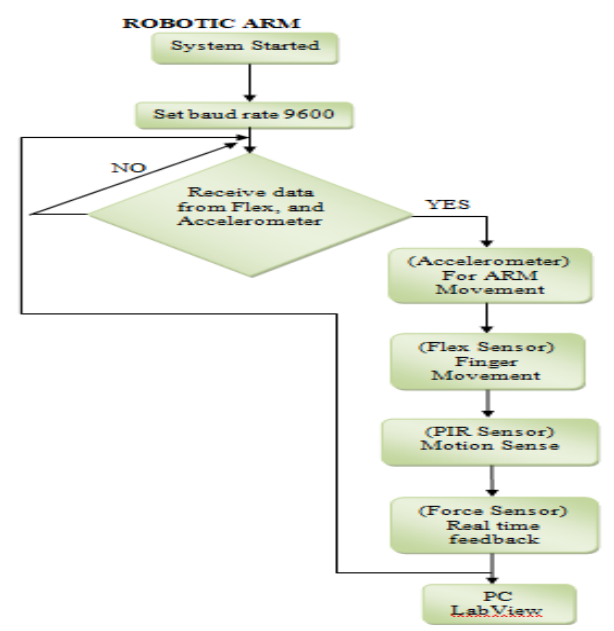

Figure 4: Flowchart of the Robotic Arm

\section{(ii) For robotic arm}

$\rightarrow$ For the operation of the robotic arm, we first initiate the system and set baud rate at $9600 \mathrm{bits} / \mathrm{sec}$.

$\rightarrow$ If the data is received from Flex and accelerometer, then the arm movements are performed using accelerometer sensor and gripper movements are performed using flex sensor.

$\rightarrow$ The PIR sensor is used for the motion detection.

$\rightarrow$ Force sensor gives the real-time feedback of the picked product.

$\rightarrow$ The controlling of the servo motors can be performed through Lab View software by serial communication.

$\rightarrow$ And the other method is to control servo motors is using through RF Zigbee.

\section{SIMULATION AND RESULTS}
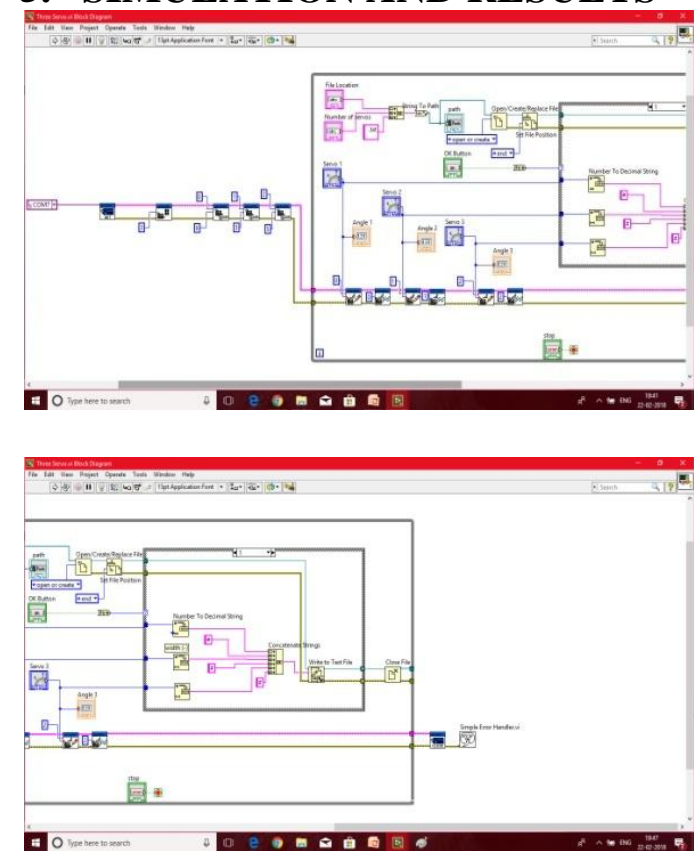

Figure 5: Block diagram panel of servo motor simulation in LabVIEW 


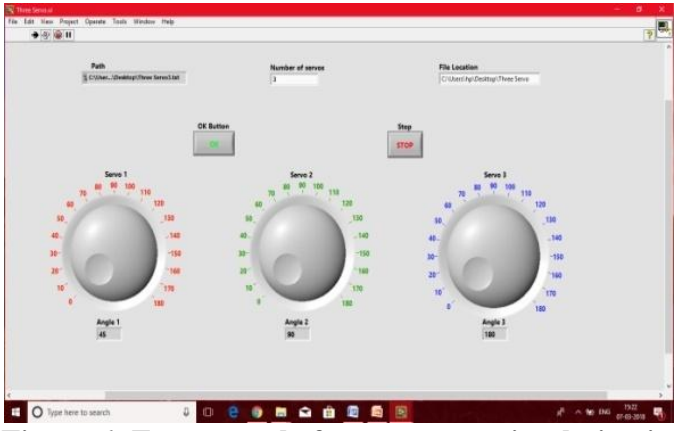

Figure 6: Front panel of servo motor simulation in LabVIEW
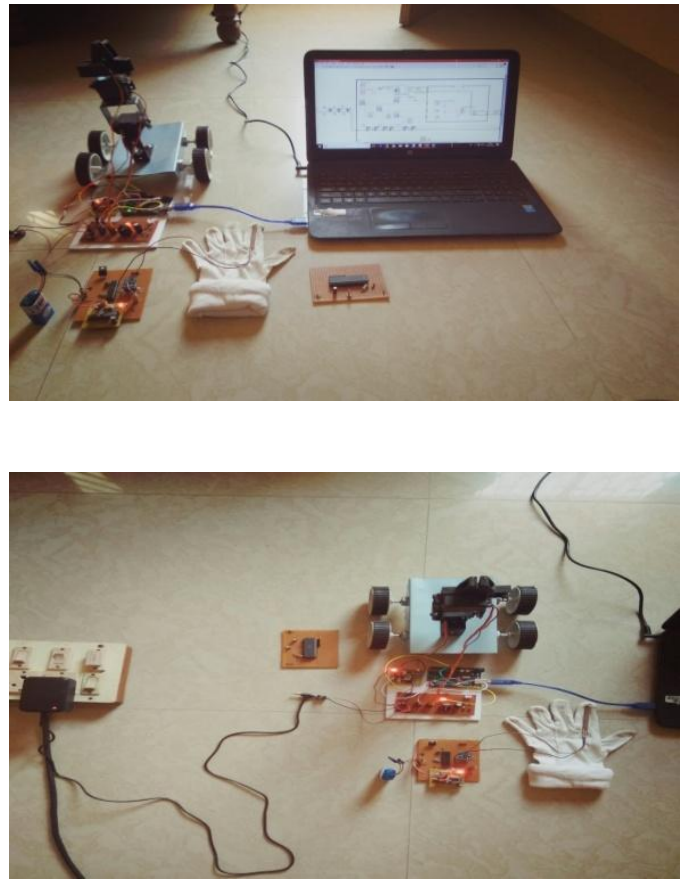

Figure 7: Working model of the simulation

\section{ADVANTAGES AND DISADVANTAGES}

\section{(i) Advantages}

$\rightarrow$ Consistent with performance

$\rightarrow$ Can be made to perform dangerous tasks without any concern

$\rightarrow$ Reduces the number of errors

$\rightarrow$ Can move from one location to another due to its wireless mechanism

$\rightarrow$ Low cost

\section{(ii) Disadvantages}

$\rightarrow$ System failure will cause breakdown

$\rightarrow$ Precise programming is needed

\section{FUTURE WORK, EXPANSION, AND CONCLUSION}

The four degrees of freedom wireless robotic arm is designed and implemented using various components in this paper. The arm is a wireless robotic arm based on LabVIEW software. The arm is controlled manually as well as semi-autonomously. The structure of arm is strengthened using aluminum brackets and servos were used as a link between joints to perform arm movements. The arm could perform various industrial tasks as well as hazardous tasks where humans can't reach easily.

The future scope of this wireless robotic arm can be implemented in industrial automation as pick and drop, as well as it could be useful in disastrous situations such as fire in a building, earthquakes, etc. in order to find human body struck under the debris and as well as in military applications.

As a part of the future expansion, the said robotic arm can be operated through other wireless modules such as WI-FI and IOT based devices. A high-definition camera could be fitted and using image processing more accurate results could be achieved.

\section{REFERENCES}

[1] Yogesh Angal, Anita Gade; "LabVIEW Controlled Robot For Object Handling Using NI myRIO";2016 IEEE International Conference on Advances in Electronics, Communication and Computer Technology (ICAECCT) Rajarshi Shahu College of Engineering, Pune India. Dec 2-3, 2016.

[2] Mr. Rahul S. Pol, Mr. Sagar Giri "LabVIEW Based Four DoF Robotic ARM"; 2016 Intl. Conference on Advances in Computing, Communications and Informatics (ICACCI), Sept. 21-24, 2016, Jaipur, India.

[3] J. Nandhini, K. Shabatini, S. Karthikeyan; "Wireless Colour Sensing Arm Robot"; International Conference on Robotics, Automation, Control and Embedded Systems - RACE 2015 18-20 February 2015, Hindustan University, Chennai, India.

[4] Liu Yunhong, Yu Haoyang, "Visual Servo Robotic Arm Control System Based on LabVIEW"; Applied Mechanics and Materials Vols. 602-605 (2014) pp 844-847.

[5] Mr. C. Chandra Mouli, Ms. P. Jyothi, Prof. K. Nagabhushan Raju, Prof. C. Nagaraja; "Design and Implementation of Robot Arm Control Using LabVIEW and ARM Controller"; IOSR Journal of Electrical and Electronics Engineering (IOSR-JEEE) e-ISSN: 2278-1676,p-ISSN: 2320-3331, Volume 6, Issue 5 (Jul. Aug. 2013), PP 80-84.

[6] Ashraf Elfasakhany, Eduardo Yanez, Karen Baylon, Ricardo Salgado; "Design and Development of a Competitive Low-Cost Robot Arm with Four Degrees of Freedom"; Modern Mechanical Engineering, 2011, 1, 47-55. 